\title{
Skilled Labor, Unskilled Labor, and Economic Growth
}

\author{
Very Preliminary version \\ Comments welcomed \\ Please do not cite
}

\author{
Marcelo Mello ${ }^{1}$
}

Faculdades Ibmec/RJ and Virginia Tech

\begin{abstract}
We assess the effects of the imperfect substitution between skilled and unskilled labor on economic growth in a model in which physical capital and skilled labor can be accumulated. It is shown that economies with higher substitutability between skilled and unskilled labor have higher levels of income per capita in the transition and in the long-run equilibrium. Furthermore, these economies have a higher level of skilled labor and a higher level of capital intensity in the long-run equilibrium. For certain parameters values, the speed of convergence depends positively on the elasticity of substitution between skilled and unskilled labor.
\end{abstract}

Keywords: Skilled Labor, Unskilled Labor, CES, Normalized production function, elasticity of substitution, Economic Growth

JEL Categories: E13, E23, O40

\footnotetext{
${ }^{1}$ Corresponding author: Mello, Department of Economics, Ibmec-RJ, Av. Rio Branco 108/1907, 20040-001, Rio de Janeiro, RJ, Brazil, ph: (5521)-4503-4161; and Department of Economics, Virginia Tech, 3016 Pamplin Hall (0316), Blacksburg, VA, 24061, USA. E-mail: mmello@ibmecri.br, and mmello@vt.edu.
} 


\section{Introduction}

While the role of the imperfect substitution between skilled and unskilled labor has been extensively analyzed in the labor economics literature, see Katz and Murphy (1992) and Autor, Katz, and Krueger (1998), for instance, little attention has been paid to this variable in the growth literature. A notable recent exception is the work of Caselli and Coleman (2006), who, in the development accounting framework, assess the role of imperfect substitution between skilled and unskilled labor in accounting for cross-country relative income. They find that higher-income countries, abundant in skilled-labor, choose technologies that best fit skilled labor, while lower-income countries, where unskilled labor is abundant, choose technologies that fit best unskilled labor ${ }^{2}$.

In this article, we study how differences in the degree of substitution between skilled and unskilled labor affect the economy in terms of its equilibrium level income per capita, capital-labor ratio, income distribution, growth rate, and its speed of income convergence. We work with a technology that is similar to the one used by Caselli and Coleman (2005). The technology has constant returns to scale in three factors of production: physical capital, skilled labor, and unskilled labor. In our model, physical capital and skilled labor can be accumulated a la Mankiw, et al. (1992). The elasticity of substitution between capital and a measure of aggregate labor is constant and equal to one, and the elasticity of substitution between skilled and unskilled labor is constant but not necessarily equal to one. The model can also be extended to include factor augmenting technological progress.

\footnotetext{
${ }^{2}$ Earlier work of Kremer and Thomson (1998), in which they study how imperfect substitution between old workers and young workers affect income convergence, could be seen as related to the topic we discuss here. In particular, the production function used by Kremer and Thomson (1998) with two inputs of production, old workers and young workers, is a CES technology similar to the ones used in the used in the labor economics where the inputs are skilled and unskilled workers.
} 
In order to examine the effects of the imperfect substitution between skilled and unskilled labor on the economy, we use the normalized production function approach, as suggested by Klump and La Grandville (2000), henceforth KG. In their article, KG, write the parameters of a CES technology in capital and labor as a function of the elasticity of substitution. They show, in the context of the Solow model, that an increase in the elasticity of substitution, all else equal, is associated with a higher capital-labor ratio and a higher level of income per capita, both in the transition and in the long-run equilibrium ${ }^{3}$.

Using the normalized production function approach we obtain a number of interesting results. First, we show that two identical economies differing only on the degree of substitution between skilled and unskilled labor, then the economy with the higher degree of substitution has a has a higher level of income per capita, a higher capital-labor ratio, and a higher proportion of skilled labor. Second, we show that two identical economies starting at the same initial equilibrium differing only on the elasticity of substitution between skilled and unskilled labor then the economy with the higher degree of substitution has, in the longrun equilibrium, a higher level of income per capita, a higher capital-labor ratio, and a higher skilled to unskilled ratio. Third, for certain parameter values, the speed of convergence depends positively on the elasticity of substitution.

Following this introduction, the next section presents the model and its main results. In section 3, an extension of the model as well as new results is presented. Section 4 is the conclusion. An appendix at the end of the article provides the derivation of the main results and equation.

\footnotetext{
${ }^{3} \mathrm{KG}$ article generated an interesting literature with several application of the normalized production function. See, for instance, Papageorgiou and Miyagiwa (2003), and Papageorgiou and Saam (2006).
} 


\section{The model}

Consider a technology with three factors of production, physical capital denoted by $K$, skilled labor denoted by $L_{s}$, and unskilled labor denoted by $L_{u}$.

$$
Y=A K^{\alpha}\left[\beta L_{u}^{\psi}+(1-\beta) L_{s}^{\psi}\right]^{(1-\alpha) / \psi}
$$

where $\mathrm{A}$ is a constant that can be interpreted as the level of the technology, $\alpha$ is the share of capital in total output, and $\beta \in(0,1)$ is the share or distribution parameter that controls the intensity in which skilled versus unskilled labor is used in the production process ${ }^{4}$, the parameter $\psi$ determines the degree of substitution between skilled and unskilled labor. More specifically, the elasticity of substitution between skilled and unskilled labor, denoted by $\sigma$, is defined as follows.

$$
\sigma=\frac{\partial\left(L_{s} / L_{u}\right)}{\partial\left(M P L_{u} / M P L_{s}\right)} \frac{M P L_{u} / M P L_{s}}{L_{s} / L_{u}}
$$

From the definition of $\sigma$, one can show that $\sigma=\frac{1}{1-\psi}$. The technology in (1) should not be viewed as representing a specific sector or firm in the economy; rather it is best interpreted in terms of the aggregate production function. Changes in the elasticity of substitution between skilled and unskilled labor should be viewed as reflecting changes in the possibilities of technical substitution between skilled and unskilled labor that come from, for example, outsourcing of labor. This interpretation is very much in line with Ventura (1997), who shows that international trade in goods, through the factor-price-equalization theorem, can make the economy's effective technology linear in capital and labor, in which case the

\footnotetext{
${ }^{4}$ The larger the coefficient $\beta$, the lower the ratio of skilled to unskilled labor for any given price ratio $w_{s} / w_{u}$, where $w_{s}$ and $w_{u}$ are, respectively, the wage rates for skilled and unskilled labor.
} 
elasticity of substitution tends towards infinite. Translating Ventura's model's result into our setting, the outsourcing of labor to cheaper overseas markets would make the domestic aggregate production function linear in skilled and unskilled labor, in which case the elasticity of substitution between skilled and unskilled labor tends towards infinite.

We assume that the total labor force, denoted by L, is constant, and it is given by the sum of skilled and unskilled labor, that is, $L=L_{u}+L_{s}$. We normalize the total labor force to the unity, and denote skilled labor by $h=L_{s} / L$; thus, unskilled labor is given by $1-h=L_{u} / L$. Equation (3) gives the production function in per capita terms.

$$
y=A k^{\alpha}\left[\beta(1-h)^{\psi}+(1-\beta) h^{\psi}\right]^{(1-\alpha) / \psi}
$$

where variables in per capita units are denoted by their corresponding lower cases. Following KG approach, we normalize the above production function (3) in terms of the elasticity of substitution between skilled and unskilled labor, that is, we write the parameters of the technology, A and $\beta$, as a function of $\sigma$. In order to do that, we choose baseline points for the marginal rate of substitution between skilled and unskilled labor, the level of income per capita, the capital-labor ratio, and the proportion of skilled labor. Denoting the baseline points by the zero subscript, we have that technical rate of substitution between skilled and unskilled labor is given by

$$
\frac{M P L_{u}}{M P L_{s}}=\frac{\beta}{1-\beta}\left(\frac{h_{0}}{1-h_{0}}\right)^{1-\psi}=\mu_{0}
$$

From equation (4), we obtain the share parameter as a function of $\sigma$ and the baseline points.

$$
\beta_{\sigma}=\frac{\mu_{0}\left(1-h_{0}\right)^{1-\psi}}{h_{0}^{1-\psi}+\mu_{0}\left(1-h_{0}\right)^{1-\psi}}
$$


Substituting equation (5) into the production function in per capita terms evaluated at the baseline point, one obtains the level of the technology, A, as a function of $\sigma$.

$$
A_{\sigma}=\frac{y_{0}}{k_{0}^{\alpha}}\left[\frac{h_{0}^{1-\psi}+\mu_{0}\left(1-h_{0}\right)^{1-\psi}}{\mu_{0}\left(1-h_{0}\right)+h_{0}}\right]^{\frac{1-\alpha}{\psi}}
$$

The normalized production function can then be written as follows.

$$
\left.y=f_{\sigma}(k, h)=A_{\sigma} k^{\alpha}\left[\beta_{\sigma}(1-h)^{\psi}+\left(1-\beta_{\sigma}\right)\right] h^{\psi}\right]^{1-\alpha}
$$

As it will be clear in section 3, the share of skilled labor in total output, defined as $\pi_{s}=\frac{M P L_{s} \cdot L_{s}}{Y}$, is a key variable in the $\operatorname{model}^{5}$. Using the normalized production function, it is easy to show that $\pi_{s}$ can be written as

$$
\pi_{s}=(1-\alpha)\left(1-\beta_{\sigma}\right) h^{\psi}\left[\frac{A_{\sigma} k^{\alpha}}{f_{\sigma}(k, h)}\right]^{\frac{\psi}{1-\alpha}}
$$

Expression (8) can be rewritten in terms of $h$ and $h_{0}$. Substituting equations (6) and (7) into equation (8), for $h \neq h_{0}, \pi_{s}$ depends on $\sigma$ through its dependency on $\psi$ (recall that $\left.\psi=\frac{\sigma-1}{\sigma}\right), h$, and $h_{0}$, according to equation (9).

$$
\pi_{s}=\frac{(1-\alpha) h_{0}^{1-\psi} h^{\psi}}{\mu_{0}\left(1-h_{0}\right)^{1-\psi}(1-h)^{\psi}+h_{0}^{1-\psi} h^{\psi}}
$$

If $h=h_{0}$, then the baseline profit share of skilled labor is given by

$$
\bar{\pi}_{s}=\frac{(1-\alpha) h_{0}}{\mu_{0}\left(1-h_{0}\right)+h_{0}}
$$

\footnotetext{
${ }^{5}$ The technology in (1) is of constant returns to scale in $K, L_{s}$, and $L_{u}$. Therefore, by Euler's theorem, we have that $1=\alpha+\pi_{s}+\pi_{u}$, where $\alpha=M P K \cdot K / Y$, and $\pi_{u}=M P L_{u} \cdot L_{u} / Y$.
} 
Note that the baseline profit share of skilled labor is independent of $\sigma$. Based on equations (9) and (10), we show in the appendix that the normalized production function can be rewritten as follows.

$$
y=y_{0}\left(\frac{k}{k_{0}}\right)^{\alpha}\left(\frac{h}{h_{0}}\right)^{1-\alpha}\left(\frac{\bar{\pi}_{s}}{\pi_{s}}\right)^{\frac{1-\alpha}{\psi}}
$$

We are now ready to establish our first result.

Theorem 1: Consider two economies, identical in every respect except in their elasticity of substitution between skilled and unskilled labor. Assume that $h \neq h_{0}$, then at any stage of the development process the economy with the higher elasticity of substitution has a higher level of income per capita.

Proof: To prove the claim, we compute the following derivative:

$$
\frac{\partial f_{\sigma}(k, h)}{\partial \sigma}=\frac{\partial f_{\sigma}}{\partial \psi} \frac{d \psi}{d \sigma}+\frac{\partial f_{\sigma}}{\partial \pi_{s}} \frac{\partial \pi_{s}}{\partial \psi} \frac{d \psi}{d \sigma}
$$

The first derivative term on the right-hand side is easy to compute. It can be obtained from the normalized production function, equation (11). It is given by

$$
\frac{\partial f_{\sigma}}{\partial \psi} \frac{d \psi}{d \sigma}=-\frac{1}{\sigma^{2}} \frac{1-\alpha}{\psi^{2}} y_{0}\left(\frac{k}{k_{0}}\right)^{\alpha}\left(\frac{h}{h_{0}}\right)^{1-\alpha}\left(\frac{\bar{\pi}_{s}}{\pi_{s}}\right)^{(1-\alpha) / \psi}\left[\ln \left(\bar{\pi}_{s} / \pi_{s}\right)\right]
$$

The second term on the right-hand side of equation (12) is difficult to compute because the derivative $\frac{\partial \pi_{s}}{\partial \psi}$ is quite cumbersome. We compute this derivative from equation (9), and we obtain the following expression:

$$
\frac{\partial \pi_{s}}{\partial \psi}=\pi_{s}\left[1-\frac{\pi_{s}}{1-\alpha}\right] \ln \left(\frac{h /(1-h)}{h_{0} /\left(1-h_{0}\right)}\right)
$$

Therefore, the second term on the right-hand side of equation (12) is given by 


$$
\frac{\partial f_{\sigma}}{\partial \pi_{s}} \frac{\partial \pi_{s}}{\partial \psi} \frac{d \psi}{d \sigma}=-\frac{1}{\sigma^{2}} \frac{1-\alpha}{\psi^{2}} y_{0}\left(\frac{k}{k_{0}}\right)^{\alpha}\left(\frac{h}{h_{0}}\right)^{1-\alpha}\left(\frac{\bar{\pi}_{s}}{\pi_{s}}\right)^{(1-\alpha) / \psi}\left[1-\frac{\pi_{s}}{1-\alpha}\right] \ln \left(\frac{h /(1-h)}{h_{0} /\left(1-h_{0}\right)}\right)
$$

Putting equations (13) and (15) together, we obtain, after some manipulation, the following expression

$$
\frac{\partial f_{\sigma}}{\partial \sigma}=-\frac{1}{\sigma^{2}} \frac{1-\alpha}{\psi^{2}} y\left[\ln \left(\frac{\bar{\pi}_{s}}{\pi_{s}}\right)+\left(1-\frac{\pi_{s}}{1-\alpha}\right) \ln \left(\frac{h /(1-h)}{h_{0} /\left(1-h_{0}\right)}\right)^{\psi}\right]
$$

Consider the second logarithmic term inside the brackets. In the appendix, we show that it can be rewritten as follows

$$
\left(\frac{h /(1-h)}{h_{0} /\left(1-h_{0}\right)}\right)^{\psi}=\left\{\frac{\pi_{s}\left[(1-\alpha)-\bar{\pi}_{s}\right]}{\bar{\pi}_{s}\left[(1-\alpha)-\pi_{s}\right]}\right\}
$$

Substituting expression in (17) into equation (16) one obtains

$$
\frac{\partial f_{\sigma}(k, h)}{\partial \sigma}=-\frac{1-\alpha}{\psi^{2}} \frac{1}{\sigma^{2}} \frac{1}{y}\left[\frac{\pi_{s}}{1-\alpha} \ln \left(\frac{\bar{\pi}_{s}}{\pi_{s}}\right)+\frac{(1-\alpha)-\pi_{s}}{1-\alpha} \ln \left(\frac{(1-\alpha)-\bar{\pi}_{s}}{(1-\alpha)-\pi_{s}}\right)\right]
$$

In order to determine the sign of the derivative in equation (18), we follow KG and use the property of strict concavity of the logarithm function. For a strict concave function, its tangent line lies above the function itself. In particular, we have that $\ln x<\ln x_{0}+\frac{\left(x-x_{0}\right)}{x_{0}}$, for a given point $x_{0}$. If we choose $x_{0}=1$, then we have that $\ln x<x-1$. Using the strict concavity of the logarithm function we have that

$$
\begin{aligned}
& \ln \left(\frac{\bar{\pi}_{s}}{\pi_{s}}\right)<\frac{\bar{\pi}_{s}}{\pi_{s}}-1 \\
& \ln \left(\frac{(1-\alpha)-\bar{\pi}_{s}}{(1-\alpha)-\pi_{s}}\right)<\frac{(1-\alpha)-\bar{\pi}_{s}}{(1-\alpha)-\pi_{s}}-1
\end{aligned}
$$


Multiplying both sides of equation (19) by $\pi_{s} /(1-\alpha)$, and multiplying both sides of equation (20) by $\frac{(1-\alpha)-\pi_{s}}{(1-\alpha)}$, we obtain the following pair of equations.

$$
\begin{aligned}
& \frac{\pi_{s}}{1-\alpha} \ln \left(\frac{\bar{\pi}_{s}}{\pi_{s}}\right)<\frac{\bar{\pi}_{s}-\pi_{s}}{1-\alpha} \\
& \frac{(1-\alpha)-\pi_{s}}{1-\alpha} \ln \left(\frac{(1-\alpha)-\bar{\pi}_{s}}{(1-\alpha)-\pi_{s}}\right)<\frac{\pi_{s}-\bar{\pi}_{s}}{(1-\alpha)}
\end{aligned}
$$

Adding up equations (21) and (22), we have that

$$
\frac{\pi_{s}}{1-\alpha} \ln \left(\frac{\bar{\pi}_{s}}{\pi_{s}}\right)+\frac{(1-\alpha)-\pi_{s}}{1-\alpha} \ln \left(\frac{(1-\alpha)-\bar{\pi}_{s}}{(1-\alpha)-\pi_{s}}\right)<0
$$

which shows that the term inside the brackets in equation (18) is negative. Thus, the sign of the derivative, $\frac{\partial f_{\sigma}(k, h)}{\partial \sigma}$, is unambiguously positive.

Papageorgiou and Saam (2006) and Dupuy and Grip (2006) derive the same result in the context of the two-level CES technology. In this case, however, it is relatively easy to prove that an increase in $\sigma$ increases the level of income, because the normalization of the two-level CES makes theorem 1 in KG, in which they show that increases in the elasticity of substitution are associated with higher income levels in the context of the traditional CES in $\mathrm{K}$ and $\mathrm{L}$, directly applicable. In our case, given the functional form of the technology in (7), this is not possible. 


\section{Dynamics of Capital and Skilled Labor}

We follow Mankiw, Romer, and Weil (1992), and assume that physical capital and skilled labor accumulate according to the following dynamic equations ${ }^{6}$.

$$
\begin{aligned}
& \dot{K}=s_{k} Y-\delta K \\
& \dot{L}_{s}=s_{s} Y-\delta L_{s}
\end{aligned}
$$

Since the labor force is constant, the accumulation equations for the capital-labor ratio and the skilled to unskilled labor ratio, $k$ and $h$, respectively, are given by

$$
\begin{aligned}
& \dot{k}=s_{k} f_{\sigma}(k, h)-\delta k \\
& \dot{h}=s_{h} f_{\sigma}(k, h)-\delta h
\end{aligned}
$$

One interesting aspect of the model, which can be seen by the accumulation equation for skilled labor, is that as the proportion of skilled labor increases, the proportion of unskilled labor decreases ${ }^{7}$. This feature of the model seems to replicate the experience of the economies in capital deepening stages. For example, Young (1995) reports that for South Korea the proportion of the population with primary and secondary education in 1966 was, respectively, $42.4 \%$ and $26.5 \%$. The same figures for 1991 were, respectively, $18.5 \%$ and 75\%. Following Mankiw et al. (1992), we set $\dot{k}=\dot{h}=0$ to obtain the steady-state equilibrium. From equations (28) and (29), we have that

$$
s_{k} y_{0}\left(\frac{k^{*}}{k_{0}}\right)^{\alpha}\left(\frac{h^{*}}{h_{0}}\right)^{1-\alpha}\left(\frac{\bar{\pi}_{s}}{\pi_{s}}\right)^{\frac{1-\alpha}{\psi}}=\delta k^{*}
$$

\footnotetext{
${ }^{6}$ We assume, as in Mankiw et. al., that the depreciation rate of physical capital and skilled labor is the same.

${ }^{7}$ There are also some technical implications of this assumption. Clearly, the proportion of skilled labor cannot be greater than one. Here we ignore problems associated with bounds on $\mathrm{h}$, because the vast majority of the economies, even the industrialized ones, are not near the boundary value for $h$.
} 


$$
s_{h} y_{0}\left(\frac{k^{*}}{k_{0}}\right)^{\alpha}\left(\frac{h^{*}}{h_{0}}\right)^{1-\alpha}\left(\frac{\bar{\pi}_{s}}{\pi_{s}}\right)^{\frac{1-\alpha}{\psi}}=\delta h^{*}
$$

From equations (30) and (31), we have that $\frac{k^{*}}{h^{*}}=\frac{s_{k}}{s_{h}}$, or $h^{*}=\frac{s_{h}}{s_{k}} k^{*}$. Substituting $h^{*}$ into equation (30), and after some rearranging, we obtain an expression for the steady-state share of skilled labor.

$$
\pi_{s}^{*}=\bar{\pi}_{s}\left(\frac{y_{0}}{k_{0}^{\alpha} h_{0}^{1-\alpha}}\right)\left(\frac{s_{k}^{\alpha} s_{h}^{1-\alpha}}{\delta}\right)^{\frac{\psi}{1-\alpha}}
$$

From the steady-state condition and the normalized production function (equation 7), we obtain the steady-state values of $k$ and $h$.

$$
\begin{aligned}
& k^{*}=\left\{\frac{s_{k}^{\frac{\psi(2-\alpha)}{1-\alpha}} \beta_{\sigma} A_{\sigma}^{\frac{\psi}{1-\alpha}}}{s_{k}^{\psi} \delta^{\frac{\psi}{1-\varepsilon}}-(1-\beta) s_{h}^{\psi}\left(s_{k} A_{\sigma}\right)^{\frac{\psi}{1-\alpha}}}\right\}^{1 / \psi} \\
& h^{*}=\frac{s_{h}}{s_{k}}\left\{\frac{s_{k}^{\frac{\psi(2-\alpha)}{1-\alpha}} \beta_{\sigma} A_{\sigma}^{\frac{\psi}{1-\alpha}}}{s_{k}^{\psi} \delta^{\frac{\psi}{1-\varepsilon}}-(1-\beta) s_{h}^{\psi}\left(s_{k} A_{\sigma}\right)^{\frac{\psi}{1-\alpha}}}\right\}^{1 / \psi}
\end{aligned}
$$

Equation (33) can be rewritten as follows.

$$
h^{*}=\left\{\frac{\beta_{\sigma}}{\left(s_{k} / s_{h}\right)^{\psi}\left(\delta / s_{k} A_{\sigma}\right)^{\frac{\psi}{1-\varepsilon}}-\left(1-\beta_{\sigma}\right)}\right\}^{1 / \psi}
$$

Our goal now is to examine how the steady-state values of $k$ and $h$ vary with $\sigma$. But first we have to make expressions (32) and (34) more tractable. From equation (8), the steadystate share of skilled labor is given by 


$$
\pi_{s}^{*}=(1-\alpha)\left(1-\beta_{\sigma}\right) h^{* \psi}\left[\frac{A_{\sigma} k^{* \alpha}}{y^{*}}\right]^{\frac{\psi}{1-\alpha}}
$$

which, assuming that $h \neq h_{0}$, can be rewritten as follows

$$
\pi_{s}^{*}=(1-\alpha)\left(1-\beta_{\sigma}\right) A_{\sigma}^{\psi /(1-\alpha)}\left(\frac{s_{k}}{\delta}\right)^{\alpha \psi /(1-\alpha)}\left(\frac{s_{h}}{\delta}\right)^{\psi}
$$

Substituting equation (36) into equation (34), after some manipulations, one obtains

$$
h^{*}=\left\{\frac{\beta_{\sigma}}{1-\beta_{\sigma}} \frac{\pi_{s}^{*}}{(1-\alpha)-\pi_{s}^{*}}\right\}^{1 / \psi}
$$

Note that $\frac{\beta_{\sigma}}{1-\beta_{\sigma}}=\frac{(1-\alpha)-\bar{\pi}_{s}}{\bar{\pi}_{s}}\left(\frac{h_{0}}{1-h_{0}}\right)^{\psi}$. Substituting this last expression into equation (37), we obtain a more tractable equation for the steady-state level of skilled labor.

$$
h^{*}=\left\{\frac{(1-\alpha)-\bar{\pi}_{s}}{\bar{\pi}_{s}} \frac{\pi_{s}^{*}}{(1-\alpha)-\pi_{s}^{*}}\right\}^{1 / \psi}\left(\frac{h_{0}}{1-h_{0}}\right)
$$

We are now ready to establish our second result.

Theorem 2: Consider two economies with identical technologies and baseline points, differing only in their $\sigma$. Assuming that the level of $\sigma$ are such that a steady-state exists, then the economy with higher $\sigma$ has a higher capital-labor ratio, a higher skilled-unskilled ratio, and a higher level of income in steady-state.

Proof: We need to compute the derivative of $h^{*}$ with respect to $\sigma$. This derivative is given by

$$
\frac{\partial h^{*}}{\partial \sigma}=\frac{\partial h^{*}}{\partial \psi} \frac{d \psi}{d \sigma}
$$


The second derivative on the right-hand side, $\frac{d \psi}{d \sigma}$, is given by $\frac{d \psi}{d \sigma}=1 / \sigma^{2}$. The first derivative on the right-hand side, $\frac{\partial h^{*}}{\partial \psi}$, is quite cumbersome to compute, so we shall proceed in steps. Let $\Delta=\left\{\frac{(1-\alpha)-\bar{\pi}_{s}}{\bar{\pi}_{s}} \frac{\pi_{s}^{*}}{(1-\alpha)-\pi_{s}^{*}}\right\}$, so that we can rewrite equation (38) for $h^{*}$ as follows.

$$
h^{*}=\left(\frac{h_{0}}{1-h_{0}}\right) e^{\frac{1}{\psi} \ln \Delta}
$$

From equation (39), the derivative of $h^{*}$ with respect to $\psi$ is given by (recall that $\bar{\pi}_{s}$ is independent of $\psi$ ).

$$
\frac{\partial h^{*}}{\partial \psi}=\left(\frac{h_{0}}{1-h_{0}}\right) \Delta^{1 / \psi}\left\{-\frac{1}{\psi^{2}} \ln \Delta+\frac{1}{\psi} \frac{\partial \ln \Delta}{\partial \psi}\right\}
$$

The derivative of the second term inside the curly-brackets is given by

$$
\frac{\partial \ln \Delta}{\partial \psi}=\frac{1}{\Delta} \frac{\partial \Delta}{\partial \psi}=\frac{1}{\Delta} \frac{(1-\alpha)-\bar{\pi}_{s}}{\bar{\pi}_{s}} \frac{(1-\alpha)}{\left[(1-\alpha)-\pi_{s}^{*}\right]^{2}} \frac{\partial \pi_{s}^{*}}{\partial \psi}
$$

which can be rewritten as

$$
\frac{\partial \ln \Delta}{\partial \psi}=\frac{1}{\psi} \frac{1-\alpha}{\left[(1-\alpha)-\pi_{s}^{*}\right]} \ln \left(\frac{\pi_{s}^{*}}{\bar{\pi}_{s}}\right)
$$

Substituting equation (42) back into equation (40), one obtains

$$
\frac{\partial h^{*}}{\partial \psi}=\left(\frac{h_{0}}{1-h_{0}}\right) \Delta^{1 / \psi}\left\{-\frac{1}{\psi^{2}} \ln \Delta+\frac{1}{\psi^{2}} \frac{(1-\alpha)}{\left[(1-\alpha)-\pi_{s}^{*}\right]} \ln \left(\frac{\pi_{s}^{*}}{\bar{\pi}_{s}}\right)\right\}
$$

Rearranging equation (43), we have that 


$$
\frac{\partial h^{*}}{\partial \psi}=-\frac{1}{\psi} \frac{1}{(1-\alpha)-\pi_{s}^{*}}\left(\frac{h_{0}}{1-h_{0}}\right) \Delta^{1 / \psi}\left\{\left[(1-\alpha)-\pi_{s}^{*}\right] \ln \left(\frac{(1-\alpha)-\bar{\pi}_{s}}{(1-\alpha)-\pi_{s}^{*}}\right)+\pi_{s}^{*} \ln \left(\frac{\bar{\pi}_{s}}{\pi_{s}^{*}}\right)\right\}
$$

By the strict concavity properties of the logarithm function, (the same argument used to prove theorem 1), it can be shown that $\left\{\left[(1-\alpha)-\pi_{s}^{*}\right] \ln \left(\frac{(1-\alpha)-\bar{\pi}_{s}}{(1-\alpha)-\pi_{s}^{*}}\right)+\pi_{s}^{*} \ln \left(\frac{\bar{\pi}_{s}}{\pi_{s}^{*}}\right)\right\}<0$, which implies that $\frac{\partial h^{*}}{\partial \psi}>0$. This shows that the steady-state level of skilled labor is a positive function of $\sigma$.

Given the steady-state relationship between $k^{*}$ and $h^{*}$, namely $k^{*}=\frac{s_{k}}{s_{h}} h^{*}$, we know that $\frac{\partial k^{*}}{\partial \psi}=\frac{s_{k}}{s_{h}} \frac{\partial h^{*}}{\partial \psi}>0$, which shows that the steady-state capital-labor ratio varies positively with $\sigma$. Finally, since $y$ is a positive function of $k, h$, and $\sigma$ in the transition it must also be a positive function of $\sigma$ in steady-state.

\section{The speed of convergence}

It can be shown that the convergence equation in our model is given by:

$$
\frac{d \ln y}{d t}=-\lambda\left(\ln y-\ln y^{*}\right)
$$

where $\lambda=\left[1-\alpha-\pi_{s}+\frac{h}{1-h} \pi_{u}\right] \delta$ gives the speed of convergence. Note that the speed of convergence depends on the skilled-labor intensity, $\frac{h}{1-h}$, weighted by the share of unskilled labor in the total income, $\pi_{u}$. Changes in the share of unskilled labor, $\pi_{u}$, affect 
the share of skilled labor, $\pi_{s}$, and vice-versa. But the share of capital in total income is constant and unaffected by changes in the share of labor ${ }^{8}$. All else equal, the additional term in convergence equation, $\frac{h}{1-h} \pi_{u}$, increases the speed of convergence. The intuition is that the higher the share of unskilled labor the higher the proportion of income that goes to the input that cannot be accumulated. This speeds up convergence because diminishing returns set in faster. On the other hand, if the share of unskilled labor is zero, which corresponds to the case where $L_{u}=0$, the model becomes one of endogenous growth in which the speed of convergence is zero. If the proportion of skilled labor is zero, $h=0$, then the expression for the speed of convergence is the same as in the traditional neoclassical model with CobbDouglas technology.

Finally, we can show that if the skilled-labor intensity is less than the baseline skilled-labor intensity, $\frac{h}{1-h}<\frac{h_{0}}{1-h_{0}}$, then the speed of convergence increases with the elasticity of substitution, that is, $\partial \lambda / \partial \sigma>0$, while if $\frac{h}{1-h}>\frac{h_{0}}{1-h_{0}}$ the sign of the derivative $\partial \lambda / \partial \sigma$ is ambiguous.

\section{Effects on the skill-premium}

[To be completed]

\section{Capital-skill complementarity}

[To be completed]

\footnotetext{
${ }^{8}$ This result follows from the fact that the elasticity of substitution between capital and aggregate labor is constant equal to one. That is, the technology is a Cobb-Douglas in capital and labor if one sees labor input as an aggregate.
} 


\section{Conclusion}

In a growth model in which the production function depends on physical capital, skilled labor, and unskilled labor, we show that an increase in the elasticity of substitution between skilled and unskilled labor, all else equal, is associated with a higher level of income per capita, in the transition and in the long-run equilibrium. Furthermore, in the long-run equilibrium, the economy with higher elasticity of substitution enjoys a higher capital-labor ratio, and a higher proportion of skilled labor. The speed of convergence, for certain, parameter values, depends positively on the elasticity of substitution.

The recent literature in economic growth has stressed the role of countries' technology as the main determinant of relative income across countries (see Parente and Prescott, 2000). There are a number of factors that influence a country's technology, such as strong institutions and openness to international trade. Our findings in this article - that increases in the elasticity of substitution between skilled and unskilled labor increase income per capita -- identify one more channel through which the technology affects income levels. In this sense, it would be an interesting topic for future research to try to pinpoint empirically the determinants of the elasticity of substitution. We already conjectured above that one possible variables affecting $\sigma$ would be degree of outsourcing of labor.

The model can be extended in several dimensions. For instance, we can include factor augmenting technological change like in Caselli and Coleman (2006) who use the following technology $Y=K^{\alpha}\left[\beta\left(A_{u} L_{u}\right)^{\psi}+(1-\beta)\left(A_{s} L\right)^{\psi}\right]^{(1-\alpha) / \psi}$, where $A_{u}$ and $A_{s}$ are, respectively, the skilled labor and unskilled labor augmenting technological progress factors. Another possible interesting extension would be to explore alternative functional forms of the production function to include capital-skill complementary. Finally, the model can also 
be used to study how the skill premium, $w_{s} / w_{u}$, responds to changes in the elasticity of substitution.

\section{Appendix}

A. Derivation of equation (11): From equations (9) and (10), one obtains the expressions below.

$$
\begin{aligned}
& \frac{\pi_{s}}{\bar{\pi}_{s}}=\frac{(1-\alpha) h_{0}^{1-\psi} h^{\psi}\left[\mu_{0}\left(1-h_{0}\right)+h_{0}\right]}{(1-\alpha) h_{0}\left[\mu_{0}\left(1-h_{0}\right)^{1-\psi}(1-h)^{\psi}+h_{0}^{1-\psi} h^{\psi}\right]} \\
& \frac{1-\bar{\pi}_{s}}{1-\pi_{s}}=\frac{\mu_{0}\left(1-h_{0}\right)+\alpha h_{0}}{\mu_{0}\left(1-h_{0}\right)+h_{0}} \frac{\mu_{0}\left(1-h_{0}\right)^{1-\psi}(1-h)^{\psi}+h_{0}^{1-\psi} h^{\psi}}{\alpha h_{0}^{1-\psi} h^{\psi}+\mu_{0}\left(1-h_{0}\right)^{1-\psi}(1-h)^{\psi}}
\end{aligned}
$$

Multiplication of equation (A1) by equation (A2) results in equation (A3).

$$
\frac{\pi_{s}}{\bar{\pi}_{s}} \frac{1-\bar{\pi}_{s}}{1-\pi_{s}}=\frac{h^{\psi}}{h_{0}^{\psi}} \frac{\mu_{0}\left(1-h_{0}\right)+\alpha h_{0}}{\alpha h_{0}^{1-\psi} h^{\psi}+\mu_{0}\left(1-h_{0}\right)^{1-\psi}(1-h)^{\psi}}
$$

Substituting equations (5) and (6) into the normalized production function, we have that

$$
y=y_{0}\left(\frac{k}{k_{0}}\right)^{\alpha}\left[\frac{\mu_{0}\left(1-h_{0}\right)^{1-\psi}(1-h)^{\psi}+h_{0}^{1-\psi} h^{\psi}}{\mu_{0}\left(1-h_{0}\right)+h_{0}}\right]^{\frac{1-\alpha}{\psi}}
$$

The term inside the brackets in equation (A4) equals $\frac{1-\bar{\pi}_{s}}{1-\pi_{s}} \frac{\alpha h_{0}^{1-\psi} h^{\psi}+\mu_{0}\left(1-h_{0}\right)^{1-\psi}(1-h)^{\psi}}{\mu_{0}\left(1-h_{0}\right)+\alpha h_{0}}$, which in turn equals $\frac{\bar{\pi}_{s}}{\pi_{s}} \frac{h^{\psi}}{h_{0}^{\psi}}$ by equation (A1). Substituting this last term into equation (A4) we obtain equation (11) which gives the normalized production function in terms of $k, h$, and $\pi_{s}$, and its baseline values.

B. Derivation of equation (17): 
Equation (A3) can be rewritten as follows

$$
\frac{\pi_{s}}{\bar{\pi}_{s}} \frac{1-\bar{\pi}_{s}}{1-\pi_{s}}=\left(\frac{h /(1-h)}{h_{0} /\left(1-h_{0}\right)}\right)^{\psi} \frac{\mu_{0}+\alpha\left(h_{0} /\left(1-h_{0}\right)\right)}{\mu_{0}+\alpha(h /(1-h))^{\psi}\left(h_{0} /\left(1-h_{0}\right)\right)^{1-\psi}}
$$

From equations (9) and (10) we have that

$$
\begin{gathered}
\left(\frac{h_{0}}{1-h_{0}}\right)=\frac{\mu_{0} \bar{\pi}_{s}}{(1-\alpha)-\bar{\pi}_{s}} \\
\left(\frac{h}{1-h}\right)=\left[\frac{\mu_{0} \pi_{s}}{(1-\alpha)-\pi_{s}}\right]^{1 / \psi}\left[\frac{(1-\alpha)-\bar{\pi}_{s}}{\mu_{0} \bar{\pi}_{s}}\right]^{(1-\psi) / \psi}
\end{gathered}
$$

Substituting equations (A6) and (A7) into equation (A5) one obtains equation (17).

\section{References}

Autor, D. H., L. F. Katz, and A. B. Kruger, 1998, “Computing Inequality: Have computers change the labor market?", The Quarterly Journal of Economics, 113 (4): 1169-1213.

Caselli, F., and A. Coleman, 2006, "The world technology frontier", The American Economic Review, forthcoming.

Dupuy, A., and A. de Grip, 2006, "Elasticity of substitution and productivity, capital and skill intensity differences across firms", Economics Letters, forthcoming.

Katz, L. F., and K. M. Murphy, 1992, "Changes in Relative Wages, 1963-1987: Supply and demand factors", The Quarterly Journal of Economics, 107 (1): 35-78.

Klump, R., and O. de La Grandeville, 2000, "Economic Growth and the Elasticity of Substitution: Two theorems and some Suggestions", American Economic Review, vol. 90, no.1: 282-291. 
Klump, R., and H. Preisseler, 2000 "CES production functions and economic growth" Scandinavian Journal of Economics, 102 (1): 41-56.

Mankiw, G., and D. Romer, and P. Weil, 1992, "A contribution to the empirics of economics growth”, The Quarterly Journal of Economics, 107 (2):407-403.

Young, A., 1995, "The Tyranny of numbers: Confronting the statistical realities of the East Asian Growth experience”, The Quarterly Journal of Economics, 110 (3): 641-680.

Ventura, J. 1997, "Growth and Interdependence”, The Quarterly Journal of Economics, 112, (1): $57-84$

Papageorgiou, C. and M. Saam, 2006, "Two-Level CES production technology in the Solow and Diamond growth models", discussion paper, Louisiana State University. , and K. Miyagiwa, 2003, Elasticity of Substitution and growth: Normalized CES in the Diamond model", Economic Theory, 21:155-165.

Parente, S. and E. Prescott, 2000, Barriers to Riches, MIT press. 